\title{
Ring-opening polymerization of epoxy end-terminated poly(ethylene oxide) as a route to highly crosslinked materials with exceptional swelling behavior (II)
}

\author{
Richard M Laine,,${ }^{1,2,3 *}$ Seung Gyoo Kim, ${ }^{1}$ Jason Rush, ${ }^{2}$ Mathew Mollan, ${ }^{4}$ \\ Hei-Jen Sun ${ }^{4}$ and Mayur Lodaya ${ }^{4}$ \\ ${ }^{1}$ Department of Materials Science and Engineering, University of Michigan, Ann Arbor, MI 48109-2136, USA \\ ${ }^{2}$ Department of Chemistry, University of Michigan, Ann Arbor, MI 48109-2136, USA \\ ${ }^{3}$ Macromolecular Science and Engineering, University of Michigan, Ann Arbor, MI 48109-2136, USA \\ ${ }^{4}$ Pfizer Pharmaceutical Sciences, Ann Arbor, Michigan, USA
}

\begin{abstract}
Highly swellable poly(ethylene oxide) (PEO) gels were prepared by anionic ring-opening polymerization of diepoxy end-capped PEO $\left(3400 \mathrm{~g} \mathrm{~mol}^{-1}\right)$ and PEO $\left(8000 \mathrm{~g} \mathrm{~mol}^{-1}\right)$ using dianionic glycerol and glycolic acid initiators at scales of up to $50 \mathrm{~g}$ diepoxide. The glycerol-derived materials swell to almost 20 times their mass in water. The driving force for rapid swelling appears to arise during 'crystallization', as segments between crosslinks are forced to pack under conditions that create high-energy domains within the material. Solvation and therefore swelling are driven by the release of the resulting packing energy. These observations may offer insight into methods for designing other highly swellable materials. When the polyfunctional initiators have groups with different reactivities (e.g. alkoxide versus carboxylate), as might be expected, the more nucleophilic functional group appears to dominate the ring-opening polymerization process.
\end{abstract}

(C) 2007 Society of Chemical Industry

Keywords: highly crosslinked polyethylene oxide; superabsorbant; ring-opening polymerization; disintegrant

\section{INTRODUCTION}

In a previous paper, we discussed model studies targeting the development of new drug delivery systems by adding functionality to poly(ethylene oxide)s (PEOs). Our objective is to develop highly crosslinked PEOs that offer novel hydrogel behavior and unusually rapid drug delivery. ${ }^{1-15}$ To do this effectively, we developed several design criteria that include:

(1) identifying functionality that provides access to tailored, hydrophilic and highly water-miscible (swellable) PEO derivatives;

(2) synthesizing derivatives with the requisite functionality using inexpensive methods;

(3) developing standardized crosslinking/polymerization reactions and characterization methods that permit comparison of properties with commercial materials;

(4) ensuring that innocuous (non-toxic) chain-linkers result following polymerization (the 'innocuous' nature of the initiator needs to be proven with toxicology studies and is not a main focus of the current study); and

(5) proving that these materials once identified can be scaled easily.
In earlier studies, we determined that anionic ring-opening polymerization (ROP) of glycidyl epoxyderivatized PEOs results in glycerol-linked PEO hydrogels that swell to more than 30 times their original mass in water. ${ }^{14}$ We also scaled the synthetic procedure. Finally, thermal analysis suggests that crosslinking coupled with crystallization creates materials wherein chain-chain packing creates highenergy states that are relieved by melting (lowering $T_{\mathrm{m}}$ ) or swelling. However, these model studies were run using tert-butylOK $(t-\mathrm{BuOK})$ as the initiator. This is not considered a standard 'innocuous' material, and hence we sought initiators that: (1) are more suitable for drug delivery systems, (2) are easily prepared and scaled and (3) offer an additional opportunity to increase the degree of functionality and crosslinking.

We discuss here the use of tri- and difunctional anionic initiators based on glycerol and glycolic acid as 'core' initiator units to produce glycerol-like crosslink segments. Traditional routes to PEO-based gels have long relied on ROP of ethylene oxide; most recently this approach has been used to make star PEOs and novel hydrogels therefrom. ${ }^{16-18}$ Our current approach is quite different because it targets forming crosslinks using PEO oligomers previously functionalized at both ends with epoxide moieties. Hence our materials not

* Correspondence to: Richard M Laine, Department of Materials Science and Engineering, University of Michigan, Ann Arbor, MI 48109-2136, USA E-mail: talsdad@umich.edu

(Received 25 July 2006; revised version received 16 November 2006; accepted 22 November 2006)

Published online 28 February 2007; DOI: 10.1002/pi.2234 
only form gels but are highly crosslinked as well, which as we have noted greatly affects molecular packing in the unswelled state, which in turn drives novel swelling behavior.

\section{EXPERIMENTAL Materials}

All chemicals were bought from standard vendors and used as received or purified as specified. Tetrahydrofuran (THF) and hexane were distilled under nitrogen from benzophenone/ $\mathrm{Na}$. Acetone was used without further purification. $\mathrm{Me}_{3} \mathrm{SiCl}$ ( $\mathrm{TMSCl}$ ) and hexamethyldisilazane (HMDS) were distilled prior to use. PEOs with $M_{\mathrm{n}}$ values of 400,3400 and $8000 \mathrm{~g} \mathrm{~mol}^{-1}$ (denoted $\mathrm{PEO}_{400}, \mathrm{PEO}_{3400}$ and $\mathrm{PEO}_{8000}$, respectively), used as a raw materials, were purchased from Aldrich and used without further purification. Molecular weights and distributions were verified using SEC (see below). Sodium hydride $(\mathrm{NaH})$ and epichlorohydrin used in the epoxidation of poly(ethylene glycol) (PEG) were purchased from Aldrich and used as received without purification. Potassium hydride (30\% dispersion in oil) was purchased from Aldrich and was washed with hexane under nitrogen prior to use.-High purity glycerol was purchased from Aldrich and used without further purification. Ethylene glycol was purchased from Aldrich and distilled prior to use. Glycolic acid was used without further purification. Lactose monohydrate (Fast Flo), and croscarmellose sodium (AcDisol) used in the disintegration tablets were purchased from Foremost and FMC, respectively.

\section{Analytical techniques}

NMR analyses

All ${ }^{1} \mathrm{H}$ and ${ }^{13} \mathrm{NMR}$ analyses were done in $\mathrm{CDCl}_{3}$ or acetone- $\mathrm{d}_{6}$ and recorded using Varian INOVA 300 and 400 spectrometers. ${ }^{1} \mathrm{H}$ NMR spectra were collected at 300.0 and $400.0 \mathrm{MHz}$ using $\mathrm{CDCl}_{3}$ or acetone- $\mathrm{d}_{6}$ as internal references at 7.24 and $2.05 \mathrm{ppm}$, respectively.

\section{SEC analysis}

SEC analyses were performed using a Waters SEC system, using a Waters 410 RI detector, Waters Styragel columns (HR 0.5, 1, 3, 4) and a PL-DCU data capture unit from Polymer Laboratory. The system was calibrated using polystyrene standards obtained from Polymer Laboratory. THF was used as the eluent, at a flow rate of $1.0 \mathrm{~mL} \mathrm{~min}^{-1}$.

\section{$X R D$ analysis}

$\mathrm{XRD}$ analysis was performed using a Rigaku rotating anode goniometer (Rigaku Denki Co. Ltd, Tokyo, Japan). Powder samples $(20-40 \mathrm{mg}$ ) were packed on a glass specimen holder. XRD scans were made from $2 \theta=10^{\circ}$ to $60^{\circ}$, using a scan rate of $2^{\circ} \mathrm{min}^{-1}$ in $0.05^{\circ}$ increments, and $\mathrm{Cu} \mathrm{K} \alpha$ radiation $(1.542 \AA$ ) operating at $40 \mathrm{kV}$ and $100 \mathrm{~mA}$.
TGA/differential thermal analysis (TGA-DTA)

TGA-DTA was performed using an SDT 2960 simultaneous DTA-TGA (TA Instruments Inc., New Castle, DE). The instrument was calibrated with gold supplied by Perkin-Elmer. Measurements were performed under a continuous flow of air $(100 \mathrm{~mL}$

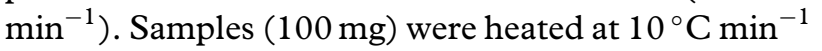
to $1400^{\circ} \mathrm{C}$ and then allowed to cool to ambient at $20^{\circ} \mathrm{C} \mathrm{min}^{-1}$. Other runs were made using a ramp rate of $10^{\circ} \mathrm{C} \mathrm{min} \min ^{-1}$ to $850^{\circ} \mathrm{C}$ and then 1,2 or $5^{\circ} \mathrm{C} \mathrm{min}{ }^{-1}$ to $800^{\circ} \mathrm{C}$.

\section{DSC studies}

DSC studies were performed using a Perkin-Elmer DSC-7 differential scanning calorimeter (PerkinElmer Co., Norwalk, CT). Measurements were performed under a continuous flow of argon $(60 \mathrm{~mL}$ $\left.\mathrm{min}^{-1}\right)$. Two heating-cooling cycles at $10^{\circ} \mathrm{C} \mathrm{min}^{-1}$ were recorded for each sample unless otherwise noted.

\section{Synthetic methods}

\section{Diepoxy PEOs}

$\mathrm{PEO}_{400}, \mathrm{PEO}_{3400}$ and $\mathrm{PEO}_{8000}$ were synthesized using procedures discussed in an earlier paper. ${ }^{14}$

\section{Trifunctional glycerol-based initiator}

Into a $250 \mathrm{~mL}$ round-bottom flask, dried under vacuum with a heat gun, was added a stoichiometric amount of base $(20.56 \mathrm{mmol})(\mathrm{KH}, \mathrm{NaH}, n$-BuLi or $\mathrm{LiCH}_{3}$ ). Dry $\mathrm{KH}$ was obtained by washing with hexane under nitrogen to remove the mineral oil prior to addition. Lithium reagents were added using syringe techniques. Then $100 \mathrm{~mL}$ dry THF was added using a dry syringe. Glycerol $(0.5 \mathrm{~mL}, 6.853 \mathrm{mmol})$ was then added through a septum at room temperature (RT). The reaction was kept under a slow flow of nitrogen to avoid evaporation and allowed to stir for 6 days after which a known concentration was used to calculate the amount needed for a ROP. For deprotonation studies, $1 \mathrm{~mL}$ samples were taken daily. The samples were capped with 9 equivalents of either HMDS or TMSCl. For selected experiments, stoichiometric $N, N$-tetramethylethylenediamine was added to improve the reactivity of the lithiating agents.

\section{Difunctional glycolic acid-based initiator}

Into a $100 \mathrm{~mL}$ round-bottom flask, dried under vacuum using a heat gun, was added a stoichiometric amount of oil-free $\mathrm{KH}(2.665 \mathrm{ml}, 19.99 \mathrm{mmol})$ cleaned as described above. To this was added $50 \mathrm{~mL}$ dry THF. In a separate $250 \mathrm{~mL}$ dry round-bottom flask was added $0.76 \mathrm{~g}(9.99 \mathrm{mmol})$ of glycolic acid. Then, $25 \mathrm{~mL}$ of dry THF was added. The solution was then stirred until the solid dissolved. The KH/THF suspension was transferred to the second flask using cannula techniques and the resulting reaction mixture was allowed to stir at RT for 6 days. During the 6 days, the appearance of the reaction mixture changed from a dark gray, granular suspension, to a white suspension of fine particles. To analyze the degree of 
deprotonation, daily samples were taken $(5 \mathrm{~mL})$ and capped with 6 eqiv. of TMSCl, $\mathrm{HMDS}$ or $\mathrm{CH}_{3} \mathrm{I}$. After 6 days no further reaction was observed.

\section{Difunctional ethylene glycol-based initiator}

Into a $250 \mathrm{~mL}$ round-bottom flask, dried under vacuum using a heat gun, was added a stoichiometric amount of oil-free $\mathrm{KH}(3.59 \mathrm{~g}, 26.93 \mathrm{mmol})$. To this was added $100 \mathrm{~mL}$ dry THF via a syringe. Then, $0.75 \mathrm{~mL}(13.46 \mathrm{mmol})$ of ethylene glycol was added via a syringe through a septum at room temperature under nitrogen and the mixture allowed to stir at RT for 6 days. A white flaky suspension formed that differed from the dark gray granular $\mathrm{KH}$ suspension. The known concentration of initiator was used to determine the volume necessary for ROP experiments.

ROP of diepoxy-PEO $\mathrm{O}_{400}$ using glycerol trianion initiator Into a vacuum/heat-dried system consisting of a $100 \mathrm{~mL}$ round-bottom flask with a magnetic stir bar and reflux condenser, $2.0 \mathrm{~g}(3.92 \mathrm{mmol})$ of diepoxy$\mathrm{PEO}_{400}$ was added under nitrogen. Dry THF was then added via a syringe to provide a final volume of $15.0 \mathrm{~mL}$. This was then heated to $70^{\circ} \mathrm{C}$ to dissolve the diepoxy-PEO 400 . Using known concentrations of initiator, $0.25,0.75,1.00$ or 1.25 equivalents were then added. For certain experiments, the total volume of initiator was diluted 100 or $200 \%$ and added at varying times.

Samples $(1.0 \mathrm{~mL})$ of reaction solution were removed by syringe at $0.5,1,2,5,10$ and $20 \mathrm{~h}$ and placed in nitrogen-filled $10 \mathrm{~mL}$ vials containing a stir bar. Then, 1.2 equivalents of $\left(\mathrm{Me}_{3} \mathrm{Si}\right)_{2} \mathrm{NH}$ was added and the mixture allowed to react with stirring at RT for $30 \mathrm{~min}$. The resulting reaction solution was added dropwise to a vial containing 10 times the amount of hexane to terminate the reaction and precipitate product. The polymer precipitates were collected by decantation and rinsed three times with hexane, and vacuum dried for longer than $2 \mathrm{~h}$, sealed and stored at $0{ }^{\circ} \mathrm{C}$. The product texture varied, depending on the equivalents of glycerol trianion and the amount of time elapsed. Final samples were vacuum dried and stored in a desiccator for swelling experiments. Molecular weight and polydispersity (PD) changes as a function of time were analyzed using SEC.

\section{ROP of diepoxy-PEO 3400 using a glycerol initiators}

Into a vacuum/heat-dried reaction system consisting of a $100 \mathrm{~mL}$ round-bottom flask with a magnetic stir bar and reflux condenser, $3.4 \mathrm{~g}(0.969 \mathrm{mmol})$ of diepoxy- $\mathrm{PEO}_{3400}$ was added under nitrogen. Dry THF was then syringed in to give a known final volume. The reaction volume was varied from 3.46 to $20 \mathrm{~mL}$ to observe volume effects on gelation. This was then heated to $70^{\circ} \mathrm{C}$ to dissolve the diepoxy-PEO. Sufficient initiator solution (prepared as above) was added to introduce $0.25,0.75,1.00$ or 1.25 equivalents to the ROP reaction solution. For certain experiments, the total volume of initiator was diluted 100 or $200 \%$ and added at varying times.

Samples $(1.0 \mathrm{~mL})$ of reaction solution were withdrawn by syringe at $0.5,1,2,5,10$ and $20 \mathrm{~h}$ and placed into a nitrogen-filled vial $(10 \mathrm{~mL})$ containing a stir bar. Then, 1.2 equivalents of $\left(\mathrm{Me}_{3} \mathrm{Si}\right)_{2} \mathrm{NH}$ was added and the mixture allowed to react with stirring at RT for $30 \mathrm{~min}$. The resulting reaction solution was then added dropwise to a vial containing 10 times the amount of hexane to terminate the reaction and precipitate the product. The polymer precipitates were collected by decantation and rinsed three times with hexane. The product was vacuum dried for longer than $2 \mathrm{~h}$, sealed and stored in a refrigerator. The product texture varied, depending on the number of equivalents of glycerol anion and the amount of time elapsed. Final samples were washed with water, vacuum dried and stored in a desiccator for swelling experiments. Molecular weight and PD changes as a function of time were analyzed using SEC.

\section{ROP of diepoxy-PEO 8000 using glycerol initiator}

Conditions and characterization methods identical to those used for diepoxy- $\mathrm{PEO}_{3400}$ were used here except that $4.0 \mathrm{~g}(0.49 \mathrm{mmol})$ of diepoxy- $\mathrm{PEO}_{8000}$ were used. Initial studies explored the effects of variations in method of preparing the initiator and types of counterions (as discussed below). The results of these studies indicated that potassium counterions provide the fastest gelation times and initiators based on potassium were then used to further optimize the gelation process.

\section{ROP of diepoxy-PEO $\mathrm{x}_{\mathrm{x}}$ using glycolic acid initiator}

Conditions and characterization methods identical to those used for diepoxy-PEO 3400 and diepoxy- $\mathrm{PEO}_{8000}$ ROP with glycerol initiator were used here. Glycolic acid initiator was prepared using $\mathrm{KH}$. Identification of optimal processing parameters in these studies (see discussion section) was used as the basis for the scaleup studies described below.

\section{Scale-up using glycerol initiator}

A $1000 \mathrm{~mL}$ round-bottom flask equipped with a magnetic stir bar and a reflux condenser was first dried under vacuum using a heat gun. After drying, the system was placed under nitrogen flow and $50 \mathrm{~g}$ diepoxy-PEO was added. After $30 \mathrm{~min}, 330 \mathrm{~mL}$ of dry $T H F$ were syringed in. The reaction mixture was then heated immediately to $70^{\circ} \mathrm{C}$. After complete dissolution, $1.00-1.25$ equivalents of glycerol initiator was added and heating was maintained for a period of 7 days resulting in a PEO gel. The obtained gel was then washed extensively with water and then vacuum dried for 7 days at $35^{\circ} \mathrm{C}$.

\section{Scale-up using glycolic acid initiator}

From the simple process optimization studies results above, scale-up studies were run to make diepoxidePEO gels. A $2000 \mathrm{~mL}$ round-bottom flask equipped 
with a mechanical stirrer and reflux condenser was first dried under vacuum using a heat gun. After drying, the system was placed under nitrogen and $50 \mathrm{~g}$ diepoxy-PEO 3400 was added. After $30 \mathrm{~min}, 330 \mathrm{~mL}$ of dry $\mathrm{THF}$ were added by syringe. The reaction mixture was then heated immediately to $70^{\circ} \mathrm{C}$. Following complete dissolution ( $c a 1 \mathrm{~h}$ ), 0.5 equivalent of the glycolic acid anion initiator was added and heating continued for 3 days to provide a PEO gel. The obtained gel was then washed with water, and vacuum dried for 3 days at $35^{\circ} \mathrm{C}$. Table 3 shows the reaction conditions and degree of swelling of PEO gels prepared with the glycolic acid initiator at $70^{\circ} \mathrm{C}$ in $\mathrm{THF}$.

\section{PEO gel swelling experiments}

These were conducted using 0.1 to $0.04 \mathrm{~g}$ of gel. Samples were cut from gelled material previously vacuum dried at room temperature and stored as described above. Gel samples were weighed and submerged in distilled water for $24 \mathrm{~h}$ at RT. The samples were then washed briefly with acetone on a Buchner funnel to remove surface water. The samples were turned during washing to ensure that all surfaces were exposed. The samples were then dried in flowing nitrogen and weighed.

\section{Disintegration in model systems}

A Spex 6800 freezer/mill was used to reduce the PEO gel particle size and the milled gel was screened using a Sonic sifter. Five percent gel with less than $150 \mu \mathrm{m}$ particle sizes was used to make $500 \mathrm{mg}$ tablets with 95\% of lactose. A Carver Laboratory Press Model C with a $3 / 8$ biconcave round punch was used to form tablets, with $5 \%$ magnesium stearate in methanol as lubricant and applied to the die prior to compaction. Tablet hardness was measured using a Distek HC97 hardness tester. Disintegration testing was performed in $900 \mathrm{~mL}$ of USP water $\left(37^{\circ} \mathrm{C}\right)$ using Pharma-Test type PTZ3EH with standard screen (size 10 mesh) basket.

\section{RESULTS AND DISCUSSION}

As expected, oxygenate anion-promoted ROP of the glycidyl diepoxides of $\mathrm{PEO}_{400}, \mathrm{PEO}_{3400}$ and $\mathrm{PEO}_{8000}$ forms glycerol-derived crosslinks. Several possible scenarios for crosslinking were envisioned per reactions (1)-(3). The diepoxy- $\mathrm{PEO}_{400}$ crosslinking studies suggest that the resulting materials probably contain, at least in part, crown ether-like segments as shown in (1). ${ }^{14}$

The objective of the work reported here was to extend the initial findings to systems that use 'innocuous' but multifunctional initiators. Although our target was formulation of the di- and trianionic species, poor solubility during formation likely results in less than complete functionalization. In fact, the data are more consistent with dianionic functionalization for the glycerol. Nonetheless, chain transfer processes allow them to function in essentially
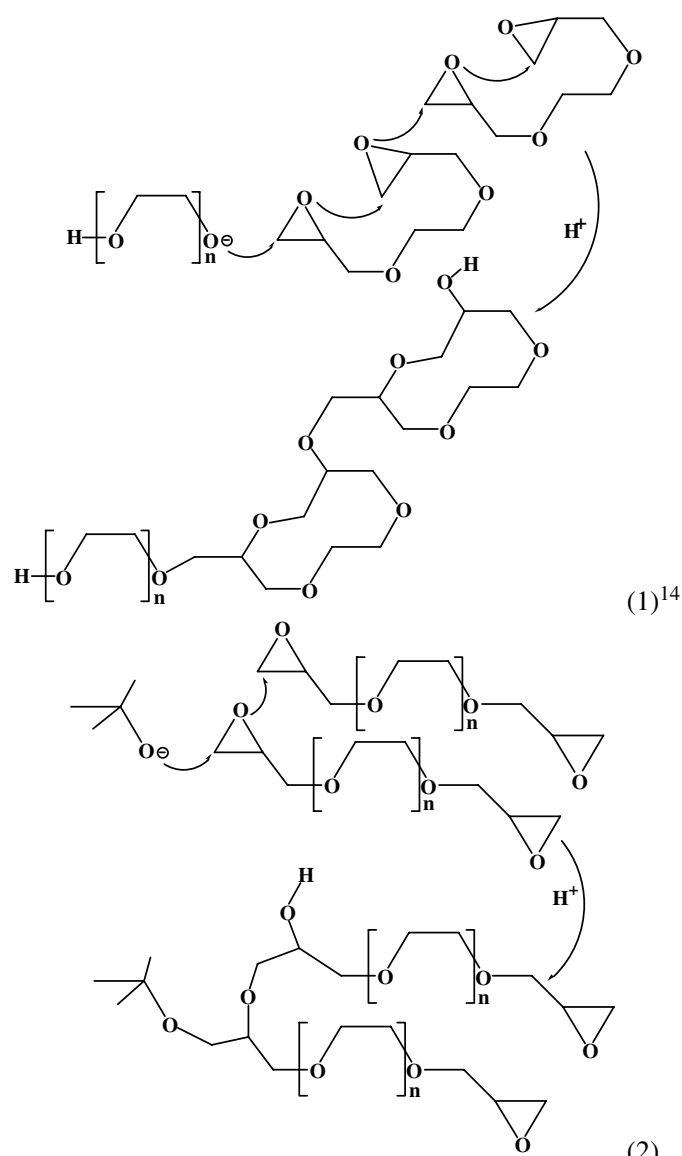

$(1)^{14}$

(2)

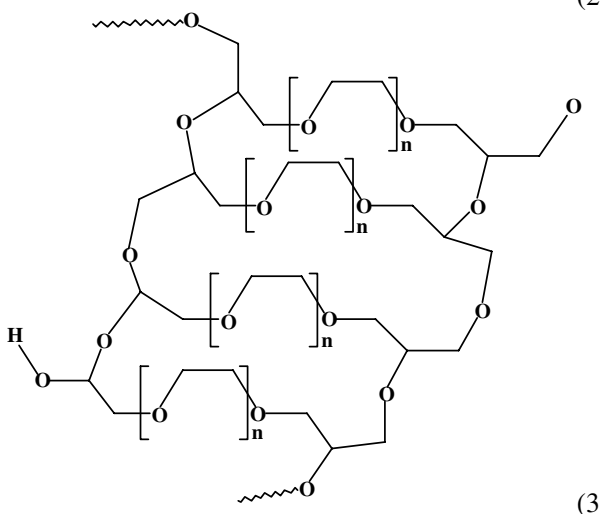

(3)

an equivalent manner to trianionic initiators, as discussed below.

In the following sections we first discuss efforts to generate and optimize formation of glycerol and glycolic acid anion initiators. We then discuss their use in forming diepoxy-PEO $\mathrm{P}_{x}$-derived hydrogels and finally we discuss the characterization of these materials.

\section{Glycerol initiator studies}

Efforts to produce the trianion of glycerol began with studies to determine the most effective route to the trianion using deprotonation. A number of potential deprotonating agents were examined under a variety of conditions as listed in Table 1. Following deprotonation under these conditions as detailed in the experimental section, the anionic species were capped with $\mathrm{Me}_{3} \mathrm{SiCl}$ or $\left(\mathrm{Me}_{3} \mathrm{Si}\right)_{2} \mathrm{NH}$ and the products analyzed using ${ }^{1} \mathrm{H}$ NMR. 
Table 1. Glycerol deprotonation studies

\begin{tabular}{|c|c|c|c|c|c|c|}
\hline \multirow[b]{2}{*}{ Trial } & \multicolumn{4}{|c|}{ Reagents used (stoichiometric amounts) } & \multicolumn{2}{|c|}{ Temperature } \\
\hline & $\mathrm{NaH}$ & $\mathrm{KH}$ & $\mathrm{LiCH}_{3}$ & TMEDA & $25^{\circ} \mathrm{C}$ & $40^{\circ} \mathrm{C}$ \\
\hline 1 & $\sqrt{ }$ & & & & $\sqrt{ }$ & \\
\hline 2 & $\sqrt{ }$ & & & & & $\sqrt{ }$ \\
\hline 3 & $\sqrt{ }$ & & & $\sqrt{ }$ & $\sqrt{ }$ & \\
\hline 4 & & $\sqrt{ }$ & & & $\sqrt{ }$ & \\
\hline 5 & & $\sqrt{ }$ & & $\sqrt{ }$ & $\sqrt{ }$ & \\
\hline 6 & & & $\sqrt{ }$ & & $\sqrt{ }$ & \\
\hline 7 & & & $\sqrt{ }$ & $\sqrt{ }$ & $\sqrt{ }$ & \\
\hline
\end{tabular}

Table 2 provides ${ }^{1} \mathrm{H}$ NMR data for glycerol and trimethylchlorosilane-capped glycerol. The ${ }^{1} \mathrm{H}$ NMR data (Table 2) indicates that the trianion does not form in significant amounts, and only dianion is observed to form. These results appear to be confirmed by the SEC data as only one peak corresponding to disilylated glycerol is observed $\left(M_{\mathrm{w}}=230 \mathrm{~g} \mathrm{~mol}^{-1}\right.$, $\left.M_{\mathrm{n}}=218 \mathrm{~g} \mathrm{~mol}^{-1}, \mathrm{PD}=1.05\right)$. However, the use of SEC for such small molecules may be unwarranted. The PD of 1.05 rather than 1.0 may arise from the fact that there are three positions that can be silylated, and hence two potential isomers are produced simultaneously.

Our inability to form the trianion could arise for several reasons, solubility and cluster formation being the most likely candidates. ${ }^{19}$ At the outset, it was determined that glycerol was not particularly soluble in any solvent where the deprotonating agents could act on it and not on the solvent. This was particularly true for $\mathrm{LiCH}_{3}$. Still another issue is the fact that alkali metal alkoxides and alkyl derivatives are known to form clusters that also can be insoluble and/or prevent full reaction with other reagents. 4,19

Most of the reaction solutions listed in Table 1 form white precipitates almost immediately on introduction of the base or glycerol to the base solvent. Although higher temperatures did accelerate the rate of dianion formation, the trianion eluded synthesis. Addition of TMEDA, known to ligate alkali metals thereby preventing or reducing cluster formation, does improve salt solubility, but never provided transparent solutions. Nonetheless, given that chain transfer was likely to give the desired products, especially where nearly complete crosslinking was expected, efforts to study ROP using the 'dianionic' glycerol species were initiated.
Preliminary studies comparing $\mathrm{K}, \mathrm{Na}$ and $\mathrm{Li}$ dianions under standard ROP conditions for diepoxy$\mathrm{PEO}_{3400}$ indicated that the lithium initiator was essentially inactive, while the sodium species showed moderate activities as expected from our original results. ${ }^{14}$ The potassium dianion was most active of all providing gelation within one day under standard conditions. These results are typical and related to the nucleophilicity of the anion, which also correlates with the size of the counterion.

Lithium, being essentially a point charge, forms clusters easily and ties up the anion very effectively. In contrast, the potassium cation is very large and does not shield the anion effectively leading to better nucleophilicity and therefore superior ROP behavior. ${ }^{4,16}$ These results are discussed in more detail below. In view of the potassium initiator results, it became the counterion of choice for the majority of the studies beyond those presented in the next section.

\section{ROP using glycerol-based initiator}

Similar to the ROP experiments conducted previously, ${ }^{14}$ diepoxy- $\mathrm{PEO}_{3400}$ was dissolved in THF, and varying amounts, 0.25 to 1.25 equivalents, of initiator were added. When $\mathrm{LiCH}_{3}$-derived initiators were added to the diepoxy- $\mathrm{PEO}_{3400}$, SEC data (not shown) did not show increases in molecular weight. ${ }^{1} \mathrm{H}$ NMR analysis indicated an insignificant degree of ring opening. As noted above, lithium anions tend to form aggregates that inhibit nucleophilicity. A standard solution is to add TMEDA to chelate the $\mathrm{Li}^{+}$ions, breaking up the aggregates. Introduction of TMEDA did indeed lead to ROP as evidenced by increases in viscosity and molecular weight. However, these rates were relatively slow and further efforts with this initiator ceased.

Given that the trianion was not detected in significant quantities, and only the dianion was formed, chain transfer assisted ROP becomes attractive. The basic process is illustrated in Fig. 1. In principle, any remaining $\mathrm{OH}$ groups will be of roughly equivalent acidity. Hence, proton exchange should be rapid leading to crosslinking processes that are essentially indistinguishable from trianionic ROP except if there are side reactions accessible by virtue of the presence of the hydroxyl groups, as suggested by Fig. 1 .

All of this assumes that proton exchange is uniform throughout the crosslinking process, which must be incorrect because segmental mobility leading to proton

Table 2. ${ }^{1} \mathrm{H}$ NMR $\left(\mathrm{CDCl}_{3}\right)$ data of glycerol and $\mathrm{KH} /$ glycerol solution after 6 days

\begin{tabular}{|c|c|c|c|c|c|c|c|}
\hline \multicolumn{4}{|c|}{ Glycerol } & \multicolumn{4}{|c|}{ Glycerol/KH capped with TMSCl } \\
\hline ppm & Integration & Mult. & Assignment & ppm & Integration & Mult. & Assignment \\
\hline 3.73 & 4 & m & $\mathrm{CH}_{2}$ & 3.73 & 1 & $\mathrm{~m}$ & $\mathrm{CH}_{2}$ \\
\hline \multirow[t]{4}{*}{3.58} & 1 & M & $\mathrm{CH}$ & 3.67 & 0.5 & $\mathrm{~m}$ & $\mathrm{CH}$ \\
\hline & & & & 3.573 & 1.5 & $\mathrm{~m}$ & $\mathrm{CH}_{2}$ \\
\hline & & & & 3.48 & 0.7 & $\mathrm{~m}$ & $\mathrm{CH}$ \\
\hline & & & & 0.098 & 18 & $\mathrm{~m}$ & $\left(\mathrm{CH}_{3}\right)_{3} \mathrm{Si}-$ \\
\hline
\end{tabular}




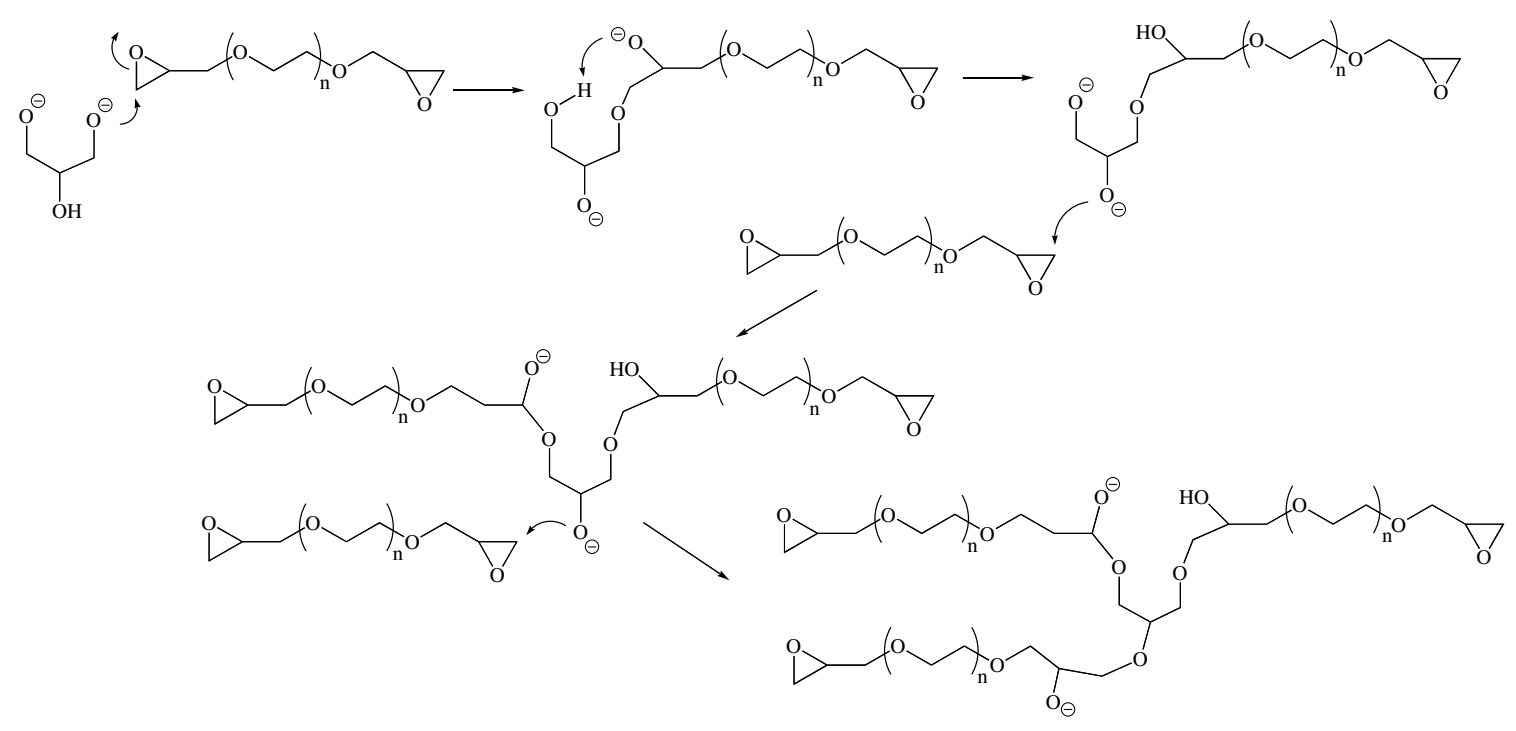

Figure 1. Chain transfer crosslinking of a diepoxy-encapped PEO using a dianionic glycerol initiator.

transfer will be retarded late in the process. In addition, depletion of epoxy groups will also reduce the overall rate of reaction. The question remains as to how uniform anion 'trapping and removal' from the ROP process is during the crosslinking process. A higher density of initiator in one region may have interesting effects on the resulting product. It may be that different cation mobilities actually control the crosslinking process at some point in the reaction. Thus the resulting product may have properties such as swelling, density and crystallinity that vary with cation type. ${ }^{16}$ Such studies remain to be undertaken; however, our goal here is to prove that highly swellable materials with innocuous initiators are accessible.

SEC studies were conducted to follow polymerization as a function of time. Unfortunately, because these materials crosslink so readily, the only products seen by SEC would be low-molecular-weight oligomers and some cyclomers. The data obtained were not useful in developing a kinetic picture of ROP of the materials because any long-chain segments would likely be crosslinked and insoluble. These data are not reported here. Thus, we rely on the properties of the products themselves to understand the polymerization process. Table 3 summarizes the types of gels produced and their swellability.
As seen in Table 3, the swelling behavior can be altered based on the concentration of the reactants and the time of initiator addition. Generally, lower concentrations and increased temporal spread of initiator addition increase swellability. Addition of 1.25 equivalents of dianion glycerol initiator gave the best results. This differs from the $t$-BuOK results where 0.75 equivalents gave optimal swellability. ${ }^{14}$ This may have to do with the chain transfer process; however, it is more likely a consequence of impurities because the scale-up studies summarized in Table 4 show swelling behaviors reminiscent of the $t$-BuOK results. ${ }^{14}$

As seen in Table 4, $50 \mathrm{~g}$ batches of diepoxide $\mathrm{PEO}_{x}$ gels were easily prepared and offered degrees of swelling of 15 to 30 times, substantiating the potential to make large quantities of these materials. Further, these materials are formed entirely from 'innocuous' initiators and crosslinkers. In the next section, we describe using glycolic acid initiator to produce diepoxy-PEO $\mathrm{PE}_{x}$ gels.

\section{Glycolic acid initiator syntheses}

Attempts to dry the glycolic acid were inhibited by its low melting point and tendency to polymerize on heating. Following dissolution in anhydrous THF and drying with $\mathrm{MgSO}_{4}$, SEC data suggest formation of a dimer as corroborated by ${ }^{1} \mathrm{H}$ NMR results, which

Table 3. Dianionic glycerol-initiated ROP of diepoxy-PEO $\mathrm{PH}_{340}$ and diepoxy- $\mathrm{PEO}_{8000}$

\begin{tabular}{|c|c|c|c|c|c|c|c|}
\hline Sample & $\begin{array}{c}\text { Volume starting } \\
\text { material/mass ratio }\left(\mathrm{mL} \mathrm{g}^{-1}\right)\end{array}$ & $\begin{array}{c}1 \text { st } \\
\text { addition } \\
\text { (equiv) }\end{array}$ & $\begin{array}{c}\text { 2nd } \\
\text { addition } \\
\text { (equiv) }\end{array}$ & $\begin{array}{c}\text { 3rd } \\
\text { addition } \\
\text { (equiv) }\end{array}$ & $\begin{array}{c}\text { Initial } \\
\text { mass (g) }\end{array}$ & $\begin{array}{c}\text { Final } \\
\text { mass (g) }\end{array}$ & $\mathrm{DS}^{\mathrm{a}}$ \\
\hline $\mathrm{PEO}_{3400}$ & 5.88 & 0.75 & 0.75 at $2 \mathrm{~h}$ & 0 & 0.116 & 0.720 & 6.6 \\
\hline $\mathrm{PEO}_{3400}$ & 3.46 & 1.25 & 0 & 0 & 0.099 & 0.745 & 7.5 \\
\hline $\mathrm{PEO}_{3400}$ & 11.76 & 1.25 & 0 & 0 & 0.107 & 1.08 & 10.1 \\
\hline $\mathrm{PEO}_{3400}$ & 4.726 & 0.416 & 0.416 at $2 \mathrm{~h}$ & 0 & 0.101 & 0.815 & 8.1 \\
\hline $\mathrm{PEO}_{3400}$ & 5.88 & 0.416 & 0.416 at $2 \mathrm{~h}$ & 0.416 at $4 \mathrm{~h}$ & 0.107 & 0.801 & 7.5 \\
\hline $\mathrm{PEO}_{8000}$ & & 0.75 & 0.5 at $4.5 h$ & 0 & 0.103 & 1.969 & 19.1 \\
\hline
\end{tabular}

${ }^{a}$ Degree of swelling. 
Table 4. Scale-up of glycerol-initiated ROP of diepoxy- $\mathrm{PEO}_{3400}$ and diepoxy- $\mathrm{PEO}_{8000}$ gels $\left(70^{\circ} \mathrm{C} / 7\right.$ days $\left./ \mathrm{THF}\right)$ and swelling behavior

\begin{tabular}{|c|c|c|c|c|c|c|}
\hline Sample & $\begin{array}{l}\text { Scale } \\
\text { (g) }\end{array}$ & $\begin{array}{l}\text { Conc. of glycerol } \\
\text { trianion (equiv) }\end{array}$ & Initial mass (g) & Final mass (g) & $\mathrm{H}_{2} \mathrm{O}$ mass $(\mathrm{g})$ & $\mathrm{DS}^{\mathrm{a}}$ \\
\hline \multirow[t]{4}{*}{ Diepoxy-PEO 3400} & 50 & 1.25 & 0.082 & 1.35 & 1.27 & 16 \\
\hline & & & 0.109 & 2.35 & 2.24 & 22 \\
\hline & & & 0.067 & 1.48 & 1.41 & 22 \\
\hline & & & 0.179 & 2.83 & 2.65 & 16 \\
\hline \multirow[t]{4}{*}{ Diepoxy-PEO 8000} & 50 & 1.25 & 0.062 & 1.91 & 1.85 & 31 \\
\hline & & & 0.045 & 0.87 & 0.83 & 19 \\
\hline & & & 0.058 & 1.56 & 1.50 & 27 \\
\hline & & & 0.079 & 1.54 & 1.46 & 20 \\
\hline
\end{tabular}

show a single peak at $4.109 \mathrm{ppm}$ suggesting formation of the dilactone. As a result, the glycolic acid used in experiments was used as received.

Based on the above-described glycerol results, most efforts focused on using the dianion made with $\mathrm{KH}$. Again, efforts to produce a well-defined anion that could be trapped and analyzed were thwarted by problems similar to those above but now compounded by the differences in acidity of the two different hydroxyl groups.

The use of a glycolic acid initiator offers some interesting diversity from the previous initiators. Perhaps the most important point is that one of the crosslinking sites will result in formation of an ester. It is possible to envision slow degradation via hydrolysis at these points in highly acidic gastric media. Hence some versions of these highly crosslinked polymers could be biodegradable.

\section{ROP using glycolic acid-based initiator}

Several gels were produced under selected conditions and their physical properties measured. Several trends are observed in the swelling behavior of these gels. As seen for gels made with other initiators, decreasing the volume and rate of initiator addition led to increased swellability. Although a wide range of initiator concentrations was used, 0.5 to 2 equivalents, a relatively small range of swelling behavior was observed. Surprisingly, when we use 0.5 equivalents
Table 5. Synthesis and swelling behavior of diepoxy-PEO $\mathrm{P}_{x}$ gels using glycolic acid initiator

\begin{tabular}{|c|c|c|c|c|c|}
\hline Sample & $\begin{array}{l}\text { Volume starting } \\
\text { material to mass } \\
\text { ratio }\left(\mathrm{mL} \mathrm{g}^{-1}\right)\end{array}$ & $\begin{array}{c}\text { 1st } \\
\text { addition } \\
\text { (equiv) }\end{array}$ & $\begin{array}{l}\text { Initial } \\
\text { mass } \\
\text { (g) }\end{array}$ & $\begin{array}{l}\text { Final } \\
\text { mass } \\
(\mathrm{g})\end{array}$ & $\mathrm{DS}^{\mathrm{a}}$ \\
\hline $\mathrm{PEO}_{3400}$ & 3 & 0.5 & 0.1 & 1.22 & 12 \\
\hline $\mathrm{PEO}_{3400}$ & 3 & 0.75 & 0.103 & 1.20 & 12 \\
\hline $\mathrm{PEO}_{3400}$ & 3 & 1 & 0.096 & 1.05 & 11 \\
\hline $\mathrm{PEO}_{3400}$ & 3.207 & 1.5 & 0.103 & 1.01 & 10 \\
\hline $\mathrm{PEO}_{3400}$ & 5.875 & 2 & 0.101 & 1.01 & 10 \\
\hline
\end{tabular}

${ }^{a} \mathrm{DS}($ degree of swelling $)=$ final mass/initial mass.

of the glycolic acid initiator, we obtain the best results, as shown in Table 5 .

Similar scale-up studies were conducted with glycolic acid initiator using the best conditions found in Table 5. Table 6 provides the data for these studies. Note that the degrees of swelling for these materials are reproducible at scale but are generally much lower than found for the $t$-BuOK and glycerol initiator studies. We suspect that the much poorer nucleophilicity of the carboxylate anion compared to the alkoxide anion is the reason for this difference. However, these results suggest that we might make the glycolic ester of the PEOs and then make the dianion of the alkoxide group in future studies:

In the scale-up studies, we used mechanical stirring in an effort to break up the gels that form during<smiles>CC(=O)COCCC(C)OC(=O)O[TlH]</smiles><smiles>CCOCC(C)OC(=O)O[AlH]</smiles>

Table 6. Synthesis conditions and degree of swelling of diepoxy- $\mathrm{PEO}_{3400}$ gels prepared by ROP with glycolic acid initiator at $70^{\circ} \mathrm{C} / 3$ days $/ \mathrm{THF}$

\begin{tabular}{lcccccc}
\hline Sample & $\begin{array}{c}\text { Scale } \\
\text { (g) }\end{array}$ & $\begin{array}{c}\text { Conc. of glycolic acid } \\
\text { (equiv) }\end{array}$ & $\begin{array}{c}\text { Initial mass } \\
(\mathrm{g})\end{array}$ & $\begin{array}{c}\text { Final mass } \\
(\mathrm{g})\end{array}$ & $\begin{array}{c}\mathrm{H}_{2} \mathrm{O} \text { mass } \\
(\mathrm{g})\end{array}$ & $\mathrm{DS}^{\mathrm{a}}$ \\
\hline Diepoxy-PEO 3400 & 50 & 0.5 & 2.8 & 31.2 & 28.4 & 11 \\
& & & 2.4 & 28.5 & 26.1 & 12 \\
& & & 2.8 & 33.2 & 30.4 & 12 \\
& & & 2.5 & 29.4 & 26.9 & 12 \\
& & & 2.2 & 23.3 & 21.1 & 11 \\
\hline
\end{tabular}

${ }^{a}$ DS (degree of swelling) = final mass/initial mass. 
ROP to obtain more uniform swelling data. However, the resulting gels still swelled greatly, absorbing most of the solvent. Consequently the reaction time was limited to only 3 days. This system deserves more detailed study in the future with more solvent and perhaps higher temperatures to allow for the poorer nucleophilicity of the carboxylate anion. In the following section, we compare the physical properties of all the gels made to date.

\section{Characterization of PEO epoxy gels}

The thermal behavior of the PEO gels was assessed using DSC at $1{ }^{\circ} \mathrm{C} \mathrm{min}^{-1}$ under nitrogen. Two heating-cooling cycles were run to ensure reproducibility. Figure 2(a) shows the DSC peaks for the diepoxy$\mathrm{PEO}_{3400}$ gel using glycerol anion initiator. In the first heating cycle, the diepoxy- $\mathrm{PEO}_{3400}$ gels exhibit a melting endotherm centered at $54{ }^{\circ} \mathrm{C}$. In the subsequent cooling cycle they exhibit cooling crystallization exotherms centered at $39^{\circ} \mathrm{C}$. In the second cycle, a second endotherm is seen giving two maxima, centered at 51 and $56^{\circ} \mathrm{C}$, respectively. The double peak seen for $\mathrm{PEO}_{3400}$ is suggested to result from chain-folded lamellae as noted in a previous paper. ${ }^{14}$

Figure 2(b) shows the properties of diepoxy$\mathrm{PEO}_{8000}$ gel. $T_{\mathrm{m}}$ and $T_{\mathrm{cc}}$ (cold crystallization temperature) of diepoxy- $\mathrm{PEO}_{8000}$ gel formed using glycerol dianion initiator are 61 and $46^{\circ} \mathrm{C}$, respectively. These values are very close to those of PEO and diepoxyPEO.

Figure 3 compares the DSC data for all diepoxy$\mathrm{PEO}_{3400}$ gels and diepoxy-PEO 8000 gels except for glycolic acid-initiated materials. As noted in the previous paper, the $\Delta H_{\mathrm{m}}$ and $\Delta H_{\mathrm{cc}}$ values (Table 7) of the PEO gels decrease significantly, especially for the $\mathrm{PEO}_{8000}$ gels, which witness drops from 140 and $150\left(\mathrm{~J} \mathrm{~g}^{-1}\right.$, second cycle) to $50-70$ and $50-60\left(\mathrm{~J} \mathrm{~g}^{-1}\right)$, respectively, depending on the initiator and cycle.

One might interpret such drops to be associated with a drop in degree of crystallinity; however, there appears to be (qualitatively) not much change in the XRD peak intensities or breadth for any of these materials (Fig. 4), and as is also apparent from the presence of easily identified peaks at higher $2 \theta$ values. It would seem reasonable to be able to determine the degree of crystallization from the XRD and these DSC results; however, recall that we have no way to account for the packing energetics that could greatly affect the DSC events observed, as discussed in our previous paper. ${ }^{14}$

The key observations from the DSC results are that these materials are all highly crystalline and recrystallize easily from the 'melt'. The concept of melt here refers to domain melting rather than complete melting given that these materials are highly crosslinked. Consequently, the $T_{\mathrm{m}}$ values observed
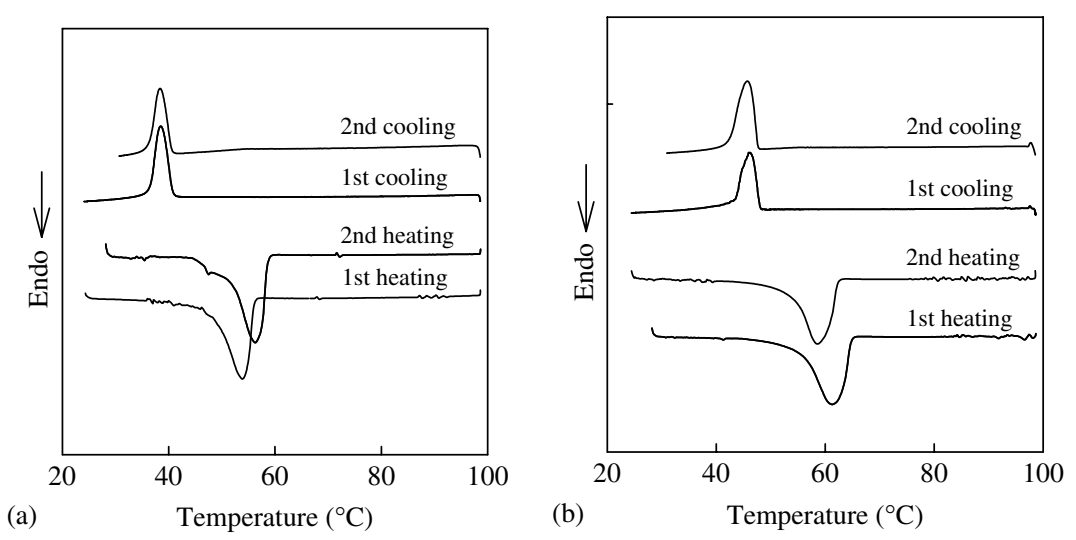

Figure 2. $\mathrm{DSC}$ curves of (a) diepoxy- $\mathrm{PEO}_{3400}$ and (b) diepoxy- $\mathrm{PEO}_{8000} \mathrm{ROP}$ gels made using glycerol potassium dianion initiator.
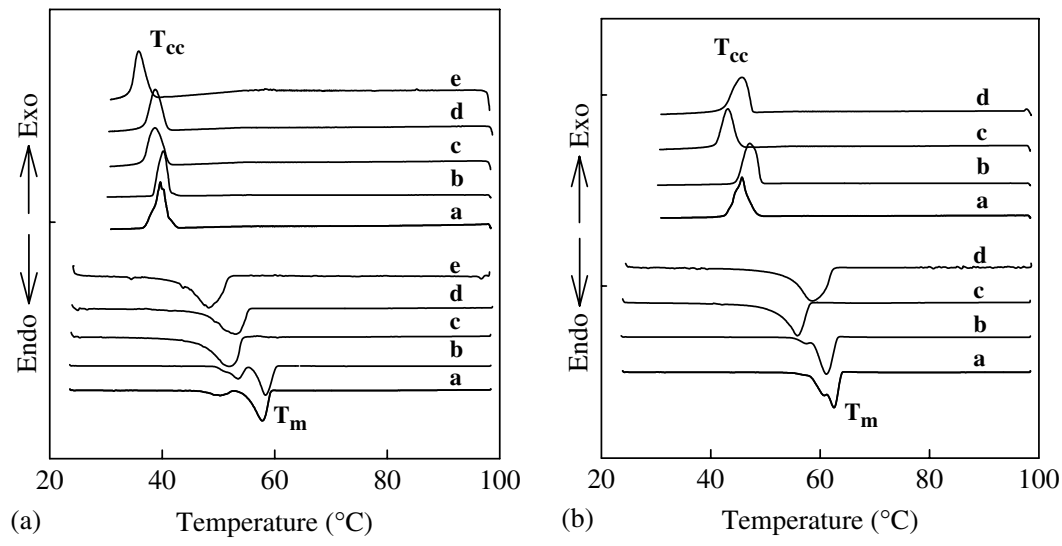

Figure 3. DSC curvess of (a) diepoxy- $\mathrm{PEO}_{3400}$ and (b) diepoxy-PEO $\mathrm{P}_{8000}$ ROP gels. a, PEG; b, diepoxy-PEO, and diepoxy-PEO gels prepared using: c, $t$-BuOK; d, glycerol; or e, glycolic acid initiators. 
Table 7. Thermal properties of PEOs, diepoxy-PEOs and diepoxy-PEO gels $\left(\mathrm{DSC} / \mathrm{N}_{2}\right.$ at $\left.1{ }^{\circ} \mathrm{C} \mathrm{min}^{-1}\right)$

\begin{tabular}{|c|c|c|c|c|c|c|c|c|c|}
\hline & & \multicolumn{4}{|c|}{ 1st scan } & \multicolumn{4}{|c|}{ 2nd scan } \\
\hline & & $\begin{array}{c}T_{\mathrm{m}} \\
\left({ }^{\circ} \mathrm{C}\right)\end{array}$ & $\begin{array}{c}\Delta H_{\mathrm{m}} \\
\left(\mathrm{J} \mathrm{g}^{-1}\right)\end{array}$ & $T_{\mathrm{CC}}\left({ }^{\circ} \mathrm{C}\right)$ & $\begin{array}{c}\Delta H_{\mathrm{fc}} \\
\left(\mathrm{J} \mathrm{g}^{-1}\right)\end{array}$ & $T_{\mathrm{m}}\left({ }^{\circ} \mathrm{C}\right)$ & $\begin{array}{c}\Delta H_{\mathrm{m}} \\
\left(\mathrm{J} \mathrm{g}^{-1}\right)\end{array}$ & $T_{\mathrm{Cc}}\left({ }^{\circ} \mathrm{C}\right)$ & $\begin{array}{c}\Delta H_{\mathrm{fc}} \\
\left(\mathrm{J} \mathrm{g}^{-1}\right)\end{array}$ \\
\hline \multirow[t]{2}{*}{ PEO } & 3400 & 58 & 186 & 41 & 164 & 58 & 182 & 40 & 156 \\
\hline & 8000 & 61 & 136 & 46 & 140 & 63 & 155 & 46 & 142 \\
\hline \multirow[t]{2}{*}{ Diepoxy-PEO } & 3400 & 54 & 68 & 39 & 60 & 52 & 65 & 39 & 61 \\
\hline & 8000 & 61 & 128 & 48 & 145 & 60 & 149 & 47 & 145 \\
\hline \multirow[t]{3}{*}{ Diepoxy-PEO 3400 gel } & $t$-BuOK & 54 & 68 & 39 & 60 & 52 & 65 & 39 & 61 \\
\hline & Glycerol & 54 & 49 & 38 & 56 & 56 & 72 & 38 & 53 \\
\hline & Glycolic acid & 48 & 58 & 36 & 57 & 49 & 59 & 36 & 56 \\
\hline \multirow[t]{2}{*}{ Diepoxy-PEO8000 gel } & $t$-BuOK & 58 & 88 & 43 & 68 & 56 & 74 & 43 & 68 \\
\hline & Glycerol & 61 & 97 & 46 & 74 & 59 & 95 & 46 & 77 \\
\hline
\end{tabular}
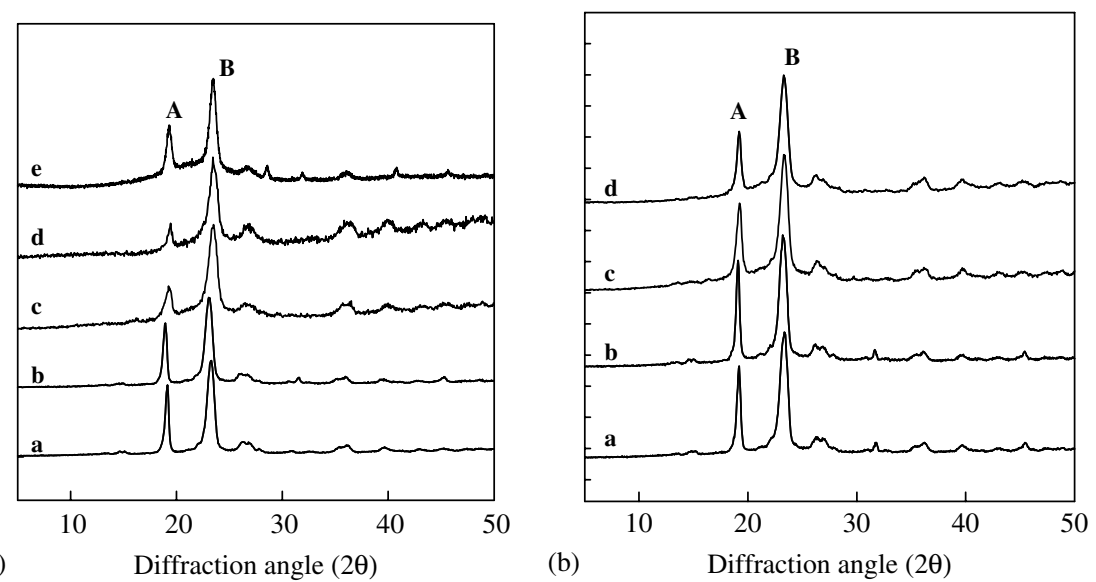

Figure 4. XRD patterns for (a) diepoxy-PEO 3400 and (b) diepoxy-PEO 8000 ROP gels. a, PEG; b, diepoxy-PEO; and diepoxy-PEO gels prepared using: c, $t$-BuOK; d, glycerol; and e, glycolic acid initiators.

must represent extensive segmental motion equivalent to domain melting rather than true melting. However as seen in our previous paper, ${ }^{14}$ the melting and crystallization enthalpies provide more detailed insight into the behavior of these materials, likely related to swelling behavior.

If the degree of crystallization is similar, then the drop in $\Delta H_{\mathrm{m}}$ and $\Delta H_{\mathrm{cc}}$ must reflect changes in energy absorbed and released during the melting and crystallization processes. If less energy is required to 'melt' the gels than in the linear PEOs, then one can argue that the crosslinked material is higher in energy in its crystallized form. Consequently, less energy input is needed to cause melting, and likewise crystallization will release less energy. This then becomes the basis for the argument that these materials swell rapidly and easily because hydration of the crosslink segments will be driven by reductions in segment-segment interactions that result in a higher energy crystalline material.

On this basis, we might expect that $\Delta H_{\mathrm{m}}$ and $\Delta H_{\mathrm{cc}}$ for the $\mathrm{PEO}_{8000}$ gels to be higher than for the $\mathrm{PEO}_{3400}$ gels because the segment lengths between crosslinks will be longer, limiting the number of crosslink-induced segment-segment interactions. This is indeed observed for the $\mathrm{PEO}_{8000}$ gels (Table 7).
Figure 4 shows XRD patterns of all of $\mathrm{PEO}_{3400}$ and $\mathrm{PEO}_{8000}$ gel materials. As predicted from the DSC data, the PEO gels were crystalline. All of the materials exhibit the same peaks regardless of the initiators, and $2 \theta, d$-spacing and full width at half maximum (FWHM) are all essentially the same. The resulting $2 \theta$ values, $d$-spacings and FWHM for these materials are essentially the same as seen previously. ${ }^{14}$ From the wide-angle XRD patterns and data, it appears that the microstructures of the domains in the diepoxy-PEO gels are unaffected by the crosslinking conditions.

\section{Disintegrations in model systems}

The large-scale $\mathrm{PEO}_{3400}$ and $\mathrm{PEO}_{8000}$ gels made with glycerol initiators were evaluated for their disintegration properties. Tablets made with PEO gels (5\%) and water-soluble lactose (95\%) were compared with tablets made with croscarmellose sodium, a standard pharmaceutical disintegrant. Compaction pressures of $10-20 \mathrm{MPa}$ were used to obtain tablet hardnesses between 12 and $15 \mathrm{kp}$. The results are listed in Table 8 and represented in Fig. 5. Lactose tablets made with $\mathrm{PEO}_{3400}$ and $\mathrm{PEO}_{8000}$ gels showed disintegration times that were at least 30\% faster than tablets made with croscarmellose sodium. Differences in tablet hardness and gel microstructures may 
Table 8. Formulation and disintegration of lactose and dicalcium phosphate tablets

\begin{tabular}{lccc}
\hline & $\begin{array}{c}\text { Pressure } \\
\text { Formulation }\end{array}$ & $\begin{array}{c}\text { Hardness } \\
(\mathrm{kp})\end{array}$ & $\begin{array}{c}\text { Disintegration } \\
\text { time } \\
(\mathrm{s})\end{array}$ \\
\hline Lactose (95\%) & 1500 & 14.5 & 61 \\
Croscarmellose & & & 62 \\
sodium (5\%) & & & 68 \\
Lactose (95\%) & 2000 & 14.7 & 42 \\
PEO 3400 gel (5\%) & & & 41 \\
& & & 42 \\
Lactose (95\%) & 1500 & 11.7 & 31 \\
PEO 8000 gel (5\%) & & & 30 \\
& & & 35 \\
\hline
\end{tabular}

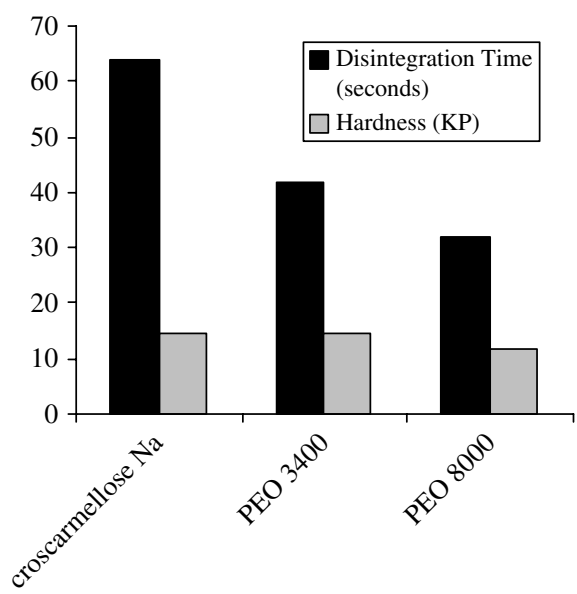

Figure 5. Disintegration time and hardness for lactose tablets $(n=3)$ made with croscarmellose sodium, $\mathrm{PEO}_{3400}$ and $\mathrm{PEO}_{8000}$ gels.

contribute to the slight differences in $\mathrm{PEO}_{8000}$ and $\mathrm{PEO}_{3400}$ tablet disintegration time.

\section{CONCLUSIONS}

The results discussed above indicate that the use of 'innocuous' polyfunctional initiators to promote ROP of diepoxide-endcapped PEOs provides access to highly swellable materials of potential use as disintegrants for drug delivery. Disintegration of lactose tablets made with PEO gels demonstrated that these polymers have superior disintegration properties than those of croscarmellose. It appears that there is a relationship between the crosslink density and the degree and rate of swelling. Thus, shorter PEO segments (e.g. 3400 versus $8000 \mathrm{~g} \mathrm{~mol}^{-1}$ ) provide more rapid swelling; unfortunately, tablet disintegration results did not show this. The mechanism for swelling is believed to arise because the shorter segments are forced to pack under conditions that create higher energy materials. Solvation and therefore swelling are driven by the release of this packing energy. These observations may offer insight into methods for designing other highly swellable materials. It appears that when the polyfunctional initiators have groups with different reactivities (e.g. alkoxide versus carboxylate), the more nucleophilic functional group dominates the ROP process.

\section{REFERENCES}

1 El-Egaky MA, Soliva M and Speiser P, Pharm Acta Helv 46:31 (1972).

2 Ford JL, Pharm Acta Helv 61:69 (1986).

3 Goertz H-H, Klinesch RG, Laemmerhirt K, Long S, Sanner A and Spengler R, Preparation of solid pharmaceutical forms. US Patent 4801460 (1989).

4 Harris JM and Zalipsky S (eds), Poly(ethylene glycol): Chemistry and Biological Applications. American Chemical Society, Washington, DC (1997).

5 Nagasaki Y and Kataoka K, ACS Polym Prepr 39:190 (1998).

6 Reutere H, Horing S and Ulbricht J, Eur Polym f 11:1113 (1989).

7 Mosquer M, Chevalier Y, Le Perchec P and Guicquero J-P, Macromol Chem Phys 198:2457 (1997).

8 Quirk RP, Lizarraga G, Lu L-M, Hasegawa H and Zhuo Q, Macromol Symp 118:89 (1997).

9 Quirk RP (ed.), Applications of Anionic Polymerization Research. American Chemical Society, Washington, DC (1998).

10 Chamow SM, Kogan TP, Venuti M, Gadek T, Haris RJ, Peers DH, et al, Bioconjugate Chem 5:133 (1994).

11 Brown CA, Synthesis 427 (1974).

12 Laine RM, Choi J and Lee I, Adv Mater 13:800 (2001).

13 Choi J, Harcup J, Yee AF, Zhu Q and Laine RM, f Am Chem Soc 123:11420 (2001).

14 Laine RM, Kim SG, Rush J, Wong E, Tamaki R, Mollan M, et al, Macromolecules 37:4525 (2004).

15 Elliott BJ, Scranton AB, Cameron JH and Bowman CN, Chem Mater 12:633 (2000).

16 Comanita B, Noren B and Roovers J, Macromolecules 32:1069 (1999).

17 Knischka R, Lutz PJ, Sunder A, Mulhaupt R and Frey H, Macromolecules 33:315 (2000).

18 Keys KB, Andreopoulos FM and Peppas NA, Macromolecules 31:8149 (1998).

19 Sigwalt P and Boileau S, $\mathcal{F}$ Polym Sci Polym Symp 62:51 (1978). 